\title{
On the Estimation of Spectral Functionals of the Fourth Order for Stationary Random Fields
}

\author{
Lyudmyla Sakhno
}

Taras Shevchenko National University of Kyiv

\begin{abstract}
This paper is concerned with the estimation of integral functionals of the fourth order spectral densities for stationary random fields based on tapered data. The considered functionals are related, in particular, to the problem of parameter estimation in the spectral domain. The main focus is on the bias of the proposed estimators, conditions for their consistency are also discussed.
\end{abstract}

Keywords: spectral estimation, higher-order spectral densities, stationary random fields, bias, tapered data.

\section{Introduction}

This paper is concerned with developing the methods for estimation of random fields in the spectral domain based on the higher-order structure of the fields. Note that higherorder statistics methods are relevant especially and essentially in those contexts where to the considered models of random processes and fields such properties pertain as nonlinearity and/or non-Gaussianity, and in all other situations where the use of the information on the second order dependence structure is not sufficient for statistical inference.

Let $X(t), t \in I$, be a real-valued measurable strictly stationary zero-mean random field, where $I$ is $\mathbb{R}^{d}$ or $Z^{d}$ endowed with the measure $\nu(\cdot)$ which is the Lebesgue or the counting measure $(\nu(\{t\})=1)$ respectively. Suppose that the field has spectral densities of order $k=2,3, \ldots$, that is, there exist the functions $f_{k}\left(\lambda_{1}, \ldots, \lambda_{k-1}\right) \in L_{1}\left(\mathbb{S}^{k-1}\right)$ such that the cumulant function of $k$-th order is given by

$$
c_{k}\left(t_{1}, \ldots, t_{k-1}\right)=\int_{\mathbb{S}^{k-1}} f_{k}\left(\lambda_{1}, \ldots, \lambda_{k-1}\right) e^{i \sum_{1}^{k-1}\left(\lambda_{j}, t_{j}\right)} d \lambda_{1} \ldots d \lambda_{k-1},
$$

where $\mathbb{S}=\mathbb{R}^{d}$ or $(-\pi, \pi]^{d}$ for the continuous and discrete cases respectively.

Let the field $X(t)$ be observed over the domain $D_{T}=[-T, T]^{d} \subset I$.

We will consider the problem of estimation of the integrals of the (cumulant) spectral densities of the fourth order:

$$
J_{4}(\varphi)=\int_{\mathbb{S}^{3}} \varphi\left(\lambda_{1}, \lambda_{2}, \lambda_{3}\right) f_{4}\left(\lambda_{1}, \lambda_{2}, \lambda_{3}\right) d \lambda_{1} d \lambda_{2} d \lambda_{3}
$$


and, as the auxiliary results, we will also study the estimates for the following integrals of the second order spectral densities:

$$
\begin{gathered}
J_{2}(\varphi)=\int_{\mathbb{S}^{2}} \varphi\left(\lambda_{1}, \lambda_{2}\right) f_{2}\left(\lambda_{1}\right) f_{2}\left(\lambda_{2}\right) d \lambda_{1} d \lambda_{2}, \\
\widehat{J}_{2}(\varphi)=\int_{\mathbb{S}} \varphi(\lambda) f_{2}^{2}(\lambda) d \lambda
\end{gathered}
$$

for the functions $\varphi$, which are appropriate for each particular integral (1)-(3).

Note that integrals (1) appear, in particular, in the problems of parameter estimation in the spectral domain within the minimum contrast (or quasi-likelihood) method developed in Anh, Leonenko, and Sakhno (2004), Anh, Leonenko, and Sakhno (2007b), where estimators are based on the Ibragimov functional constructed with the use of information on higher-order spectral densities. The integrals (3) appear in the expressions for covariance matrices of the asymptotic normal law for the Whittle estimators for Gaussian processes and fields, as well as for the Ibragimov estimators based on the second order spectra.

We study the estimators for the functionals (1)-(3) based on tapered periodograms introduced below and mainly focussing on bias of the considered estimators. The use of data tapers leads to the bias reduction, which is especially important for spatial data: tapers can help to attenuate the so-called "edge effects" (see, e.g. Dahlhaus and Künsch (1987), Guyon (1995)). Consider the tapered values

$$
\left\{h_{T}(t) X(t), t \in D_{T}\right\},
$$

where $h_{T}(t)=h(t / T), t=\left(t^{(1)}, \ldots, t^{(d)}\right) \in \mathbb{R}^{d}$, and the taper $h(t)$ factorizes as $h(t)=$ $\prod_{i=1}^{d} \tilde{h}\left(t^{(i)}\right), t^{(i)} \in \mathbb{R}^{1}$, with $\tilde{h}(\cdot)$ satisfying the assumption below.

Assumption 1. $\tilde{h}(t), t \in \mathbb{R}$, is a positive measurable even function of bounded variation with bounded support: $\tilde{h}(t)=0$ for $|t|>1$.

Denote

$$
\tilde{H}_{k, T}(\lambda)=\int \tilde{h}_{T}(t)^{k} e^{-i \lambda t} \nu(d t) \quad \text { and } \quad H_{k, T}(\lambda)=\int h_{T}(t)^{k} e^{-i(\lambda, t)} \nu(d t)=\prod_{i=1}^{d} \tilde{H}_{k, T}\left(\lambda^{(i)}\right) .
$$

The integrals above are one-dimensional and $d$-dimensional with corresponding measure $\nu(\cdot)$ (for the discrete case we have sums).

Define the finite Fourier transform of tapered data $\left\{h_{T}(t) X(t), t \in D_{T}\right\}$ :

$$
d_{T}^{h}(\lambda)=\int h_{T}(t) X(t) e^{-i(\lambda, t)} \nu(d t), \lambda \in \mathbb{S},
$$

the tapered periodograms of the second and the third orders:

$$
\begin{gathered}
I_{2, T}^{h}(\lambda)=\frac{1}{(2 \pi)^{d} H_{2, T}(0)} d_{T}^{h}(\lambda) d_{T}^{h}(-\lambda), \\
I_{3, T}^{h}\left(\lambda_{1}, \lambda_{2}\right)=\frac{1}{(2 \pi)^{2 d} H_{3, T}(0)} d_{T}^{h}\left(\lambda_{1}\right) d_{T}^{h}\left(\lambda_{2}\right) d_{T}^{h}\left(-\lambda_{1}-\lambda_{2}\right)
\end{gathered}
$$

(provided that $H_{2, T}(0) \neq 0$ and $H_{3, T}(0) \neq 0$ correspondingly),

and the tapered periodogram of $k$-th order (provided that $H_{k, T}(0) \neq 0$ ):

$$
I_{k, T}^{h}\left(\lambda_{1}, \ldots, \lambda_{k-1}\right)=\frac{1}{(2 \pi)^{(k-1) d} H_{k, T}(0)} \prod_{i=1}^{k} d_{T}^{h}\left(\lambda_{i}\right), \quad \lambda_{i} \in \mathbb{S},
$$

where $\sum_{i=1}^{k} \lambda_{i}=0$, but no proper subset of $\lambda_{i}$ has sum 0 (that is, there is no other subset $\left\{\lambda_{i}, i \in \nu\right\}$, where $\nu=\left\{i_{1}, \ldots, i_{l}\right\} \subset\{1, \ldots, k\}$ and $\sum_{i \in \nu} \lambda_{i}=0$, these subsets are called 
submanifolds (following, e.g., Brillinger and Rosenblatt (1967)). Denote the set of such submanifolds by $\Lambda_{k}$.

The statistic (6) is a natural generalization of the second-order periodogram and can serve as an estimator for the spectral density of $k$-th order $f_{k}\left(\lambda_{1}, \ldots, \lambda_{k-1}\right)$ at all frequencies but except those from $\Lambda_{k}$. Consistent estimators of $f_{k}$ can be obtained by taking weighted averages of values (6) at the neighborhood of frequencies of interest, avoiding points from $\Lambda_{k}$.

Therefore, the problem of estimations of the integrals

$$
J_{k}(\varphi)=\int_{\mathbb{S}^{k-1}} \varphi_{k}(\lambda) f_{k}(\lambda) d \lambda
$$

can be solved by the following approaches:

1) let the function $\psi\left(\lambda_{1}, \ldots, \lambda_{k}\right)$ defined on $\left\{\lambda_{i}: \sum_{i=1}^{k} \lambda_{i}=0\right\}$ be such that $\psi\left(\lambda_{1}, \ldots, \lambda_{k}\right) \equiv 0$ for $\lambda=\left(\lambda_{1}, \ldots, \lambda_{k}\right) \in \Lambda_{k}$, then one can consider the empirical spectral functional

$$
\int_{\mathbb{S}^{k-1}} \varphi_{k}(\lambda) I_{k, T}^{h}(\lambda) \psi(\lambda) d \lambda
$$

as the estimate for the integral

$$
\int_{\mathbb{S}^{k-1}} \varphi_{k}(\lambda) f_{k}(\lambda) \psi(\lambda) d \lambda
$$

(see, e.g., Anh et al. (2004), Anh et al. (2007b));

2) another way to exclude from consideration the points from $\Lambda_{k}$ is to consider the functional

$$
J_{k, T}^{*}\left(\varphi_{k}\right)=J_{k, T, \varepsilon}^{*}\left(\varphi_{k}\right)=\int_{S_{\varepsilon}^{k-1}} \varphi_{k}(\lambda) I_{k, T}^{h}(\lambda) d \lambda,
$$

as an estimate for

$$
J_{k}^{*}\left(\varphi_{k}\right)=J_{k, \varepsilon}^{*}\left(\varphi_{k}\right)=\int_{S_{\varepsilon}^{k-1}} \varphi_{k}(\lambda) f_{k}(\lambda) d \lambda,
$$

for all $\varepsilon>0$, where the integration is taken over $S^{k-1}$, but avoiding the frequencies on and neighboring to the submanifolds $\left\{\sum_{i \in \nu} \lambda_{i}=0\right.$, where $\left.\nu=\left\{i_{1}, \ldots, i_{l}\right\} \subset\{1, \ldots, k\}\right\}$. More precisely, $S_{\varepsilon}^{k-1}=S^{k-1} \backslash\left\{\lambda:\left|\sum_{i \in \nu} \lambda_{i}\right|<\varepsilon\right.$ for all $\left.\nu \subset\{1, \ldots, k\}\right\}$, where $|y|=\max _{1 \leq i \leq d}\left|y^{(i)}\right|$. This approach was used in Anh, Leonenko, and Sakhno (2010), under some conditions the statistics (11) can serve as an estimate for (7).

One more way to estimate the integral (7) is via recursion, that is, the estimate is constructed with the use of some combination of the integral of the periodogram of the $k$-th order and integrals of products of lower order periodograms. This approach was suggested in Taniguchi (1982) for the case of the integrals of the fourth order spectra and was further generalized in Keenan (1987) for the estimation of integrals of the $k$-th order spectra. Both mentioned authors considered discrete time processes and used conditions in the time domain, namely, conditions of summability of cumulants. Extension of this method to the case of fields was presented in Sakhno (2011).

In the present paper we develop the ideas of papers Taniguchi (1982), Keenan (1987), Sakhno (2011) and study the estimates for the fourth order spectral integrals (1) and integrals (2)-(3) for stationary random fields, basing on the tapered data and with conditions formulated in the spectral domain. The main results are stated in the next section and followed by some discussion. Auxiliary facts and formulas used for the proofs are relegated into Appendix.

\section{Estimation of the spectral functionals from tapered data}

Consider the problem of estimation of the functional

$$
J_{4}(\varphi)=\int_{\mathbb{S}^{3}} \varphi\left(\lambda_{1}, \lambda_{2}, \lambda_{3}\right) f_{4}\left(\lambda_{1}, \lambda_{2}, \lambda_{3}\right) d \lambda_{1} d \lambda_{2} d \lambda_{3}
$$


Introduce the empirical spectral functional obtained by substitution of the fourth order tapered periodogram $I_{4, T}^{h}$ (given by the formula (6) with $k=4$ ) instead of $f_{4}$ into (12):

$$
J_{4, T}(\varphi)=\int_{\mathbb{S}^{3}} \varphi\left(\lambda_{1}, \lambda_{2}, \lambda_{3}\right) I_{4, T}^{h}\left(\lambda_{1}, \lambda_{2}, \lambda_{3}\right) d \lambda_{1} d \lambda_{2} d \lambda_{3} .
$$

Note that we do not restrict the domain of integration and do not exclude the sets $\Lambda_{4}$ as it would be prescribed by the definition of the 4 -th order periodogram.

The expectation of the empirical functional (13) is given by

$$
\begin{aligned}
E J_{4, T}(\varphi)= & \frac{1}{(2 \pi)^{3 d} H_{4, T}(0)} \int_{\mathbb{S}^{3}} \varphi\left(\lambda_{1}, \lambda_{2}, \lambda_{3}\right) \int_{\mathbb{S}^{3}} f_{4}\left(\gamma_{1}, \gamma_{2}, \gamma_{3}\right) \\
& \times \prod_{i=1}^{3} H_{1, T}\left(\gamma_{i}-\lambda_{i}\right) H_{1, T}\left(-\sum_{i=1}^{3} \gamma_{i}+\sum_{i=1}^{3} \lambda_{i}\right) d \gamma_{1} d \gamma_{2} d \gamma_{3} d \lambda_{1} d \lambda_{2} d \lambda_{3} \\
+ & \int_{\mathbb{S}^{3}} \varphi\left(\lambda_{1}, \lambda_{2}, \lambda_{3}\right) \int_{\mathbb{S}} f_{2}\left(\gamma_{1}\right) H_{1, T}\left(\gamma_{1}-\lambda_{1}\right) H_{1, T}\left(-\gamma_{1}-\lambda_{2}\right) \\
& \times \int_{\mathbb{S}} f_{2}\left(\gamma_{2}\right) H_{1, T}\left(\gamma_{2}-\lambda_{3}\right) H_{1, T}\left(-\gamma_{2}+\sum_{i=1}^{3} \lambda_{i}\right) d \gamma_{1} d \gamma_{2} d \lambda_{1} d \lambda_{2} d \lambda_{3} \\
+ & \int_{\mathbb{S}^{3}} \varphi\left(\lambda_{1}, \lambda_{2}, \lambda_{3}\right) \int_{\mathbb{S}} f_{2}\left(\gamma_{1}\right) H_{1, T}\left(\gamma_{1}-\lambda_{1}\right) H_{1, T}\left(-\gamma_{1}-\lambda_{3}\right) \\
& \times \int_{\mathbb{S}^{3}} f_{2}\left(\gamma_{2}\right) H_{1, T}\left(\gamma_{2}-\lambda_{2}\right) H_{1, T}\left(-\gamma_{2}+\sum_{i=1}^{3} \lambda_{i}\right) d \gamma_{1} d \gamma_{2} d \lambda_{1} d \lambda_{2} d \lambda_{3} \\
+ & \int_{\mathbb{S}^{3}} \varphi\left(\lambda_{1}, \lambda_{2}, \lambda_{3}\right) \int_{\mathbb{S}^{3}} f_{2}\left(\gamma_{1}\right) H_{1, T}\left(\gamma_{1}-\lambda_{1}\right) H_{1, T}\left(-\gamma_{1}+\sum_{i=1}^{3} \lambda_{i}\right) \\
& \times \int_{\mathbb{S}} f_{2}\left(\gamma_{2}\right) H_{1, T}\left(\gamma_{2}-\lambda_{2}\right) H_{1, T}\left(-\gamma_{2}-\lambda_{3}\right) d \gamma_{1} d \gamma_{2} d \lambda_{1} d \lambda_{2} d \lambda_{3} .
\end{aligned}
$$

For calculations we used the formula for cumulants of products of random variables to obtain

$$
\begin{aligned}
E I_{4, T}^{h}\left(\lambda_{1}, \lambda_{2}, \lambda_{3}\right)= & \operatorname{cum}\left(d_{T}^{h}\left(\lambda_{1}\right), d_{T}^{h}\left(\lambda_{2}\right), d_{T}^{h}\left(\lambda_{3}\right), d_{T}^{h}\left(-\lambda_{1}-\lambda_{2}-\lambda_{3}\right)\right) \\
& +\operatorname{cum}\left(d_{T}^{h}\left(\lambda_{1}\right), d_{T}^{h}\left(\lambda_{2}\right)\right) \operatorname{cum}\left(d_{T}^{h}\left(\lambda_{3}\right), d_{T}^{h}\left(-\lambda_{1}-\lambda_{2}-\lambda_{3}\right)\right) \\
& +\operatorname{cum}\left(d_{T}^{h}\left(\lambda_{1}\right), d_{T}^{h}\left(\lambda_{3}\right)\right) \operatorname{cum}\left(d_{T}^{h}\left(\lambda_{2}\right), d_{T}^{h}\left(-\lambda_{1}-\lambda_{2}-\lambda_{3}\right)\right) \\
& +\operatorname{cum}\left(d_{T}^{h}\left(\lambda_{1}\right), d_{T}^{h}\left(-\lambda_{1}-\lambda_{2}-\lambda_{3}\right)\right) \operatorname{cum}\left(d_{T}^{h}\left(\lambda_{2}\right), d_{T}^{h}\left(\lambda_{3}\right)\right)
\end{aligned}
$$

and then we used the formula A.1 (see Appendix) for cumulants of finite Fourier transforms. We now analyze the asymptotic behavior of $E J_{4, T}(\varphi)$.

Introduce the assumptions, which will be used to state the results. In what follows we suppose that the taper satisfies Assumption 1 and for different statements we will need some additional assumptions on the taper and different assumptions on the spectral densities $f_{2}, f_{4}$ and the function $\varphi$.

Assumption 2. The taper $\tilde{h}(t)$ is a Lipschitz-continuous function on $[-1,1]$.

Assumption 3. The function $\varkappa_{2}^{h}(u)=\left|\int \tilde{h}(t) e^{-i t u} d t\right|^{2}$ satisfies:

$$
\int|u|^{l} \varkappa_{2}^{h}(u)(u) d u<\infty, l=1,2 .
$$

Assumption 4. The function $\varkappa_{4}^{h}(u)=\int \tilde{h}(t) e^{-i t u} d t \int \tilde{h}^{3}(t) e^{i t u} d t$ satisfies:

$$
\int|u|^{l} \varkappa_{3}^{h}(u)(u) d u<\infty, l=1,2 .
$$


Assumption 5. The spectral densities $f_{2}, f_{4}$ and the function $\varphi$ are continuous and for the case $\mathbb{S}=\mathbb{R}$ suppose additionally $\varphi \in L_{1} \cap L_{2}, f_{4} \in L_{2}$.

Assumption 6. The spectral density $f_{4}$ and the function $\varphi$ are twice boundedly differentiable and $\varphi \in L_{1}$.

Assumption 7. The spectral density $f_{2}$ is twice boundedly differentiable and $\varphi \in L_{1}$.

Theorem 1. I. Let the spectral densities $f_{2}, f_{4}$ and the function $\varphi$ satisfy Assumption 5. Then as $T \rightarrow \infty$

$$
\begin{aligned}
E J_{4, T}(\varphi) \rightarrow & \int_{\mathbb{S}^{3}} \varphi\left(\lambda_{1}, \lambda_{2}, \lambda_{3}\right) f_{4}\left(\lambda_{1}, \lambda_{2}, \lambda_{3}\right) d \lambda_{1} d \lambda_{2} d \lambda_{3} \\
& +\int_{\mathbb{S}^{2}}\left\{\varphi\left(\lambda_{1},-\lambda_{1}, \lambda_{2}\right)+\varphi\left(\lambda_{1}, \lambda_{2},-\lambda_{1}\right)\right. \\
& \left.\quad+\varphi\left(\lambda_{1},-\lambda_{1}, \lambda_{2}\right)\right\} f_{2}\left(\lambda_{1}\right) f_{2}\left(\lambda_{2}\right) d \lambda_{1} d \lambda_{2}=: \widetilde{J}_{4}(\varphi)
\end{aligned}
$$

II. Let the taper $\tilde{h}(t)$ satisfy Assumption 2 for the case of discrete-parameter fields, and for the case of continuous-parameter fields let Assumption 4 hold; the spectral density $f_{4}$ and the function $\varphi$ satisfy Assumption 6. Then

$$
E J_{4, T}(\varphi)-\widetilde{J}_{4}(\varphi)=O\left(T^{-2}\right) \text { as } T \rightarrow \infty .
$$

Proof. I. The expression (14) for $E J_{4, T}(\varphi)$ can be written in the form

$$
\begin{gathered}
E J_{4, T}(\varphi)=\int_{\mathbb{S}^{3}} \varphi\left(\lambda_{1}, \lambda_{2}, \lambda_{3}\right) \int_{\mathbb{S}^{3}} f_{4}\left(\gamma_{1}, \gamma_{2}, \gamma_{3}\right) \\
\times \Phi_{4, T}\left(\gamma_{1}-\lambda_{1}, \gamma_{2}-\lambda_{2}, \gamma_{3}-\lambda_{3}\right) d \gamma_{1} d \gamma_{2} d \gamma_{3} d \lambda_{1} d \lambda_{2} d \lambda_{3} \\
+\int_{\mathbb{S}^{3}} \varphi\left(\lambda_{1}, \lambda_{2}, \lambda_{3}\right) \int_{\mathbb{S}^{2}} f_{2}\left(\gamma_{1}\right) f_{2}\left(\gamma_{2}\right) \Phi_{4, T}\left(\gamma_{1}-\lambda_{1},-\gamma_{1}-\lambda_{2}, \gamma_{2}-\lambda_{3}\right) d \gamma_{1} d \gamma_{2} d \lambda_{1} d \lambda_{2} d \lambda_{3} \\
+\int_{\mathbb{S}^{3}} \varphi\left(\lambda_{1}, \lambda_{2}, \lambda_{3}\right) \int_{\mathbb{S}^{2}} f_{2}\left(\gamma_{1}\right) f_{2}\left(\gamma_{2}\right) \Phi_{4, T}\left(\gamma_{1}-\lambda_{1},-\gamma_{1}-\lambda_{3}, \gamma_{2}-\lambda_{2}\right) d \gamma_{1} d \gamma_{2} d \lambda_{1} d \lambda_{2} d \lambda_{3} \\
+\int_{\mathbb{S}^{3}} \varphi\left(\lambda_{1}, \lambda_{2}, \lambda_{3}\right) \int_{\mathbb{S}^{2}} f_{2}\left(\gamma_{1}\right) f_{2}\left(\gamma_{2}\right) \Phi_{4, T}\left(\gamma_{1}-\lambda_{1}, \gamma_{2}-\lambda_{2},-\gamma_{2}-\lambda_{3}\right) d \gamma_{1} d \gamma_{2} d \lambda_{1} d \lambda_{2} d \lambda_{3},
\end{gathered}
$$

where $\Phi_{4, T}\left(u_{1}, u_{2}, u_{3}\right)$ are the multidimensional kernels of Fourier type (see Appendix). Under the imposed assumptions on the functions $\varphi, f_{2}, f_{4}$, using the property (A.2) of the kernels, we obtain the convergence (24).

II. The proof of the second part of the theorem is obtained by the reasoning similar to those applied in Anh et al. (2010) for the proof of Theorem 2.3, where the expectation of the functional $J_{k}^{*}\left(\varphi_{k}\right)$ (see (11)) was analyzed, namely, we adapt for our needs here the first part of that proof concerned with the term containing the $k$-th order spectral density. Note that this approach generalizes that applied in Sakhno (2007) in the study of second-order spectral functionals. The main steps of the proof are presented separately for continuous and discrete cases.

Consider the continuous case $\left(\mathbb{S}=\mathbb{R}^{d}\right)$. We can rewrite the first term in the formula (14) in the following form

$$
\begin{aligned}
I_{1}= & \frac{1}{(2 \pi)^{3 d} H_{4, T}(0)} \int_{\mathbb{S}^{3}} \varphi\left(\lambda_{1}, \lambda_{2}, \lambda_{3}\right) \int_{\mathbb{S}^{3}} f_{4}\left(\lambda_{1}+u_{1}, \ldots, \lambda_{3}+u_{3}\right) \\
& \times \prod_{j=1}^{4} H_{1, T}\left(u_{j}\right) \delta\left(\sum_{j=1}^{4} u_{j}\right) d u_{1} d u_{2} d u_{3} d \lambda_{1} d \lambda_{2} d \lambda_{3},
\end{aligned}
$$


where $H_{4, T}(0)=T^{d}\left(\tilde{H}_{4}(0)\right)^{d}, H_{1, T}\left(u_{j}\right)=T^{d} \prod_{i=1}^{d} \tilde{H}_{1}\left(T u_{j}^{(i)}\right), \tilde{H}_{k}(u)=\int \tilde{h}(t)^{k} e^{-i t u} d t$.

Changing the variables $T u_{j}^{(i)}=v_{j}^{(i)}$, we obtain

$$
\begin{aligned}
I_{1}= & \frac{1}{(2 \pi)^{3 d}\left(\tilde{H}_{4}(0)\right)^{d}} \int_{\mathbb{S}^{3}} \varphi\left(\lambda_{1}, \lambda_{2}, \lambda_{3}\right) \int_{\mathbb{S}^{3}} f_{4}\left(\lambda_{1}+\frac{v_{1}}{T}, \ldots, \lambda_{3}+\frac{v_{3}}{T}\right) \\
& \times \prod_{j=1}^{4} \prod_{i=1}^{d} \tilde{H}_{1}\left(v_{j}^{(i)}\right) \delta\left(\sum_{j=1}^{4} v_{j}\right) d v_{1} d v_{2} d v_{3} d \lambda_{1} d \lambda_{2} d \lambda_{3} .
\end{aligned}
$$

Since $f_{4}$ is twice boundedly differentiable, using Taylor's theorem we can write

$$
f_{4}\left(\lambda_{1}+\frac{v_{1}}{T}, \ldots, \lambda_{3}+\frac{v_{3}}{T}\right)=f_{4}\left(\lambda_{1}, \lambda_{2}, \lambda_{3}\right)+\text { const } \cdot T^{-1} \sum_{j=1}^{3} \sum_{i=1}^{d} v_{j}^{(i)}+O\left(T^{-2}\right) \sum_{j=1}^{3} \sum_{i=1}^{d}\left|v_{j}^{(i)}\right|^{2}
$$

(uniformly in $\lambda$ in $O$-term), correspondingly, for $I_{1}$ we obtain

$$
I_{1}=\int_{\mathbb{S}^{3}} \varphi\left(\lambda_{1}, \lambda_{2}, \lambda_{3}\right) f_{4}\left(\lambda_{1}, \lambda_{2}, \lambda_{3}\right) d \lambda_{1} d \lambda_{2} d \lambda_{3}+R_{1}+R_{2} .
$$

The error term $R_{1}$ is of the form

$$
\begin{aligned}
R_{1}= & \text { const } \cdot \frac{1}{(2 \pi)^{3 d}\left(\tilde{H}_{4}(0)\right)^{d}} \int_{\mathbb{S}^{3}} \varphi\left(\lambda_{1}, \lambda_{2}, \lambda_{3}\right) \frac{1}{T} \sum_{j=1}^{3} \sum_{i=1}^{d} \int_{\mathbb{S}^{3}} v_{j}^{(i)} \\
& \times \prod_{j=1}^{4} \prod_{i=1}^{d} \tilde{H}_{1}\left(v_{j}^{(i)}\right) \delta\left(\sum_{j=1}^{4} v_{j}\right) d v_{1} d v_{2} d v_{3} d \lambda_{1} d \lambda_{2} d \lambda_{3},
\end{aligned}
$$

this reduces to the sum of terms of the form

$$
\text { const } \cdot \frac{1}{T} \int_{\mathbb{S}^{3}} \varphi\left(\lambda_{1}, \lambda_{2}, \lambda_{3}\right) \int_{\mathbb{R}} v_{j}^{(i)} \tilde{H}_{1}\left(v_{j}^{(i)}\right) \tilde{H}_{3}\left(-v_{j}^{(i)}\right) d v_{j}^{(i)} d \lambda_{1} d \lambda_{2} d \lambda_{3},
$$

each of which is equal to zero since $\tilde{H}_{1}\left(v_{j}^{(i)}\right) \tilde{H}_{3}\left(-v_{j}^{(i)}\right)$ is an even function.

Analogously, $R_{2}$ reduces to the sum of terms of the following form

$$
\text { const } \cdot \int_{\mathbb{S}^{3}} \varphi\left(\lambda_{1}, \lambda_{2}, \lambda_{3}\right) \int_{\mathbb{R}}\left|v_{j}^{(i)}\right|^{2} \tilde{H}_{1}\left(v_{j}^{(i)}\right) \tilde{H}_{3}\left(-v_{j}^{(i)}\right) d v_{j}^{(i)} d \lambda_{1} d \lambda_{2} d \lambda_{3},
$$

supplied with multiplier $O\left(T^{-2}\right)$. Due to Assumption 4 on the taper and integrability of $\varphi$, the integrals (19) are bounded, therefore

$$
I_{1}=\int_{\mathbb{S}^{3}} \varphi\left(\lambda_{1}, \lambda_{2}, \lambda_{3}\right) f_{4}\left(\lambda_{1}, \lambda_{2}, \lambda_{3}\right) d \lambda_{1} d \lambda_{2} d \lambda_{3}+O\left(T^{-2}\right) .
$$

Consider now the expression (18) for $I_{1}$ in the discrete case $\left(\mathbb{S}=(-\pi, \pi]^{d}\right)$. We again use the Taylor's theorem for the twice boundedly differentiable function $f_{4}$ and write

$$
\left|f_{4}\left(\lambda_{1}+u_{1}, \ldots, \lambda_{3}+u_{3}\right)-f_{4}\left(\lambda_{1}, \lambda_{2}, \lambda_{3}\right)-\sum_{j=1}^{3} \sum_{i=1}^{d} u_{j}^{(i)} \frac{\partial f_{4}(\lambda)}{\partial \lambda_{j}^{(i)}}\right| \leq \text { const } \cdot \sum_{j=1}^{3} \sum_{i=1}^{d}\left|u_{j}^{(i)}\right|^{2} .
$$

Denote

$$
\alpha_{T}=I_{1}-\int_{\mathbb{S}^{3}} \varphi\left(\lambda_{1}, \lambda_{2}, \lambda_{3}\right) f_{4}\left(\lambda_{1}, \lambda_{2}, \lambda_{3}\right) d \lambda_{1} d \lambda_{2} d \lambda_{3}
$$


Consider the expression

$$
\begin{aligned}
& \frac{1}{(2 \pi)^{3 d} H_{4, T}(0)} \sum_{j=1}^{3} \sum_{i=1}^{d} \int_{\mathbb{S}^{3}} \varphi\left(\lambda_{1}, \lambda_{2}, \lambda_{3}\right) \frac{\partial f_{4}(\lambda)}{\partial \lambda_{j}^{(i)}} \int_{\mathbb{S}^{3}} u_{j}^{(i)} \\
& \times \prod_{j=1}^{4} \prod_{i=1}^{d} \tilde{H}_{1, T}\left(u_{j}^{(i)}\right) \delta\left(\sum_{j=1}^{4} u_{j}\right) d u_{1} d u_{2} d u_{3} d \lambda_{1} d \lambda_{2} d \lambda_{3} .
\end{aligned}
$$

The inner integrals in (21) reduce to the expressions

$$
\left\{(2 \pi)^{(3)} \tilde{H}_{4, T}(0)\right\}^{d-1}(2 \pi)^{2} \int_{-\pi}^{\pi} u_{j}^{(i)} \tilde{H}_{1, T}\left(u_{j}^{(i)}\right) \tilde{H}_{3, T}\left(-u_{j}^{(i)}\right) d u_{j}^{(i)}
$$

and the last integral is equal to zero since $\tilde{H}_{1, T}\left(u_{j}^{(i)}\right) \tilde{H}_{3, T}\left(-u_{j}^{(i)}\right)$ is an even function. We conclude that (21) is equal to zero and

$$
\begin{aligned}
\left|\alpha_{T}\right| \leq & \text { const } \cdot \sum_{j=1}^{3} \sum_{i=1}^{d} \int_{\mathbb{S}^{3}}\left|\varphi\left(\lambda_{1}, \lambda_{2}, \lambda_{3}\right)\right|\left[\frac{1}{(2 \pi)^{3 d} H_{4, T}(0)} \int_{\mathbb{S}^{3}}\left|u_{j}^{(i)}\right|^{2}\right. \\
& \left.\times \prod_{j=1}^{4} \prod_{i=1}^{d}\left|\tilde{H}_{1, T}\left(u_{j}^{(i)}\right)\right| \delta\left(\sum_{j=1}^{4} u_{j}\right) d u_{1} d u_{2} d u_{3}\right] d \lambda_{1} d \lambda_{2} d \lambda_{3} .
\end{aligned}
$$

Using the properties of the functions $H_{k, T}$, we obtain the bound for the expression in square brackets in (22):

$$
\text { const } \cdot \frac{1}{T} \int_{-\pi}^{\pi}|u|^{2}\left|\tilde{H}_{1, T}(u) \tilde{H}_{3, T}(-u)\right| d u \text {. }
$$

Taking into account that for a Lipschitz-continuous taper $\tilde{h}(t)$

$$
\int_{-\pi}^{\pi}|u|^{2}\left|\tilde{H}_{k, T}(u)\right|^{2} d u \leq \frac{1}{T}
$$

we conclude that each term in the left hand side of $(22)$ is bounded by const $\cdot T^{-2}$, therefore, we have for $I_{1}$ the same asymptotics (20) as in the continuous case.

The terms from the second to the fourth in the formula (14) can be analysed in the similar manner: we repeat the above steps but taking now the Taylor expansions for the function $\varphi$. In such a way we can show that the sum of these terms converges to the second term in the sum in the l.h.s. of (24) with the same rate $O\left(T^{-2}\right)$. This completes the proof.

From the expression $(24)$ we conclude that to obtain the estimate for the functional $J_{4}(\varphi)$ we should subtract from $J_{4, T}(\varphi)$ the estimate for the functinal appearing as the second term in the sum in the l.h.s. of (24), that is, we need the estimates for the functionals $J_{2}(\varphi)$ given by (2). To this aim, consider the empirical spectral functional

$$
J_{2, T}(\varphi)=\int_{\mathbb{S}^{2}} \varphi\left(\lambda_{1}, \lambda_{2}\right) I_{2, T}\left(\lambda_{1}\right) I_{2, T}\left(\lambda_{2}\right) d \lambda_{1} d \lambda_{2} .
$$

Theorem 2. I. Let the spectral densities $f_{2}, f_{4}$ and the function $\varphi$ satisfy Assumption 5. Then as $T \rightarrow \infty$

$$
E J_{2, T}(\varphi) \rightarrow J_{2}(\varphi)=\int_{\mathbb{S}^{2}} \varphi\left(\lambda_{1}, \lambda_{2}\right) f_{2}\left(\lambda_{1}\right) f_{2}\left(\lambda_{2}\right) d \lambda_{1} d \lambda_{2}
$$

II. Let the taper $\tilde{h}(t)$ satisfy Assumption 2 for the case of discrete-parameter fields, and for the case of continuous-parameter fields let Assumption 3 hold; the spectral density $f_{2}$ and the function $\varphi$ satisfy Assumption \%. Then

$$
E J_{2, T}(\varphi)-J_{2}(\varphi)=O\left(T^{-2}\right) \text { as } T \rightarrow \infty .
$$


Proof. Analogously to calculation of $E J_{4, T}(\varphi)$, we obtain:

$$
\begin{aligned}
& E J_{2, T}(\varphi)=\frac{1}{\left((2 \pi)^{3 d} H_{2, T}(0)\right)^{2}} \int_{\mathbb{S}^{2}} \varphi\left(\lambda_{1}, \lambda_{2}\right)\left\{\int_{\mathbb{S}^{3}} f_{4}\left(\gamma_{1}, \gamma_{2}, \gamma_{3}\right) H_{1, T}\left(\gamma_{1}-\lambda_{1}\right)\right. \\
& \times H_{1, T}\left(\gamma_{2}+\lambda_{1}\right) H_{1, T}\left(\gamma_{3}-\lambda_{2}\right) H_{1, T}\left(-\sum_{i=1}^{3} \gamma_{i}+\lambda_{2}\right) d \gamma_{1} d \gamma_{2} d \gamma_{3} \\
& +\int_{\mathbb{S}} f_{2}\left(\gamma_{1}\right) H_{1, T}\left(\gamma_{1}-\lambda_{1}\right) H_{1, T}\left(-\gamma_{1}+\lambda_{1}\right) d \gamma_{1} \int_{\mathbb{S}} f_{2}\left(\gamma_{2}\right) H_{1, T}\left(\gamma_{2}-\lambda_{2}\right) H_{1, T}\left(-\gamma_{2}+\lambda_{2}\right) d \gamma_{2} \\
& +\int_{\mathbb{S}} f_{2}\left(\gamma_{1}\right) H_{1, T}\left(\gamma_{1}-\lambda_{1}\right) H_{1, T}\left(-\gamma_{1}-\lambda_{2}\right) d \gamma_{1} \int_{\mathbb{S}} f_{2}\left(\gamma_{2}\right) H_{1, T}\left(\gamma_{2}+\lambda_{1}\right) H_{1, T}\left(-\gamma_{2}+\lambda_{2}\right) d \gamma_{2} \\
& +\int_{\mathbb{S}} f_{2}\left(\gamma_{1}\right) H_{1, T}\left(\gamma_{1}-\lambda_{1}\right) H_{1, T}\left(-\gamma_{1}+\lambda_{2}\right) \\
& \left.\times \int_{\mathbb{S}} f_{2}\left(\gamma_{2}\right) H_{1, T}\left(\gamma_{2}+\lambda_{1}\right) H_{1, T}\left(-\gamma_{2}-\lambda_{2}\right) d \gamma_{1} d \gamma_{2}\right\} d \lambda_{1} d \lambda_{2},
\end{aligned}
$$

which can be also written in the following form:

$$
\begin{aligned}
& C_{T}\left[\int_{\mathbb{S}^{2}} \varphi\left(\lambda_{1}, \lambda_{2}\right) \int_{\mathbb{S}^{2}} f_{4}\left(\gamma_{1}, \gamma_{2}, \gamma_{3}\right) \Phi_{4, T}\left(\gamma_{1}-\lambda_{1}, \gamma_{2}+\lambda_{1}, \gamma_{3}-\lambda_{2}\right) d \gamma_{1} d \gamma_{2} d \lambda_{1} d \lambda_{2}\right. \\
& +\int_{\mathbb{S}^{2}} \varphi\left(\lambda_{1}, \lambda_{2}\right) \int_{\mathbb{S}^{2}} f_{2}\left(\gamma_{1}\right) f_{2}\left(\gamma_{2}\right) \Phi_{4, T}\left(\gamma_{1}-\lambda_{1},-\gamma_{1}-\lambda_{2}, \gamma_{2}+\lambda_{1}\right) d \gamma_{1} d \gamma_{2} d \lambda_{1} d \lambda_{2} \\
& \left.+\int_{\mathbb{S}^{2}} \varphi\left(\lambda_{1}, \lambda_{2}\right) \int_{\mathbb{S}^{2}} f_{2}\left(\gamma_{1}\right) f_{2}\left(\gamma_{2}\right) \Phi_{4, T}\left(\gamma_{1}-\lambda_{1},-\gamma_{1}+\lambda_{2}, \gamma_{2}+\lambda_{1}\right) d \gamma_{1} d \gamma_{2} d \lambda_{1} d \lambda_{2}\right] \\
& +\int_{\mathbb{S}^{2}} \varphi\left(\lambda_{1}, \lambda_{2}\right) \int_{\mathbb{S}} f_{2}\left(\gamma_{1}\right) \Phi_{2, T}\left(\gamma_{1}-\lambda_{1}\right) d \gamma_{1} \int_{\mathbb{S}} f_{2}\left(\gamma_{2}\right) \Phi_{2, T}\left(\gamma_{2}-\lambda_{2}\right) d \gamma_{2} d \lambda_{1} d \lambda_{2},
\end{aligned}
$$

where we have denoted

$$
C_{T}=H_{4, T}(0)\left((2 \pi)^{d} H_{2, T}(0)\right)^{-2} .
$$

Considering (27), taking into account the properties of the kernels $\Phi_{2, T}$ and $\Phi_{4, T}$ (see (A.2))and the conditions imposed on the functions $\varphi, f_{2}$ and $f_{4}$ in the first part of the theorem, we can see that the last integral in (27) converges to $J_{2}(\varphi)$ as $T \rightarrow \infty$, the expression in the square brackets converges to the finite limit, but being supplied with the factor $C_{T}$ (which is of order $\frac{1}{T}$ ) this part of the sum vanishes. To evaluate the rate of convergence of bias for the second part of the theorem we use the similar reasonings as those in the proof of Theorem 1, we need to analyze actually only the last term in (27) to obtain the statement.

From Theorem 2 we have an immediate consequence concerning estimation of the functional $\widehat{J}_{2}(\varphi)$ given by $(3)$. Consider the functional

$$
\widehat{J_{2, T}}(\varphi)=\frac{1}{2} \int_{\mathbb{S}} \varphi(\lambda)\left(I_{2, T}(\lambda)\right)^{2} d \lambda
$$

Theorem 3. $I$. Let the spectral densities $f_{2}, f_{4}$ and the function $\varphi$ satisfy Assumption 5. Then as $T \rightarrow \infty$

$$
E \widehat{J_{2, T}}(\varphi) \rightarrow \widehat{J_{2}}(\varphi)
$$

II. Let the taper $\tilde{h}(t)$ satisfy Assumption 2 for the case of discrete-parameter fields, and for the case of continuous-parameter fields let Assumption 3 hold; the spectral density $f_{2}$ and the function $\varphi$ satisfy Assumption 7. Then

$$
E \widehat{J_{2, T}}(\varphi)-\widehat{J_{2}}(\varphi)=O\left(T^{-2}\right) \text { as } T \rightarrow \infty
$$


Introduce the following empirical functional

$$
\begin{aligned}
\widehat{J_{4, T}}(\varphi)= & J_{4, T}(\varphi)-\int_{\mathbb{S}^{2}} I_{2, T}\left(\lambda_{1}\right) I_{2, T}\left(\lambda_{2}\right) \\
& \times\left\{\varphi\left(\lambda_{1},-\lambda_{1}, \lambda_{2}\right)+\varphi\left(\lambda_{1}, \lambda_{2},-\lambda_{1}\right)+\varphi\left(\lambda_{1},-\lambda_{1}, \lambda_{2}\right)\right\} d \lambda_{1} d \lambda_{2} .
\end{aligned}
$$

As a straightforward corollary of Theorems 1 and 2 we obtain the next result.

Theorem 4. I. Let the taper, spectral densities $f_{2}, f_{4}$ and the function $\varphi$ satisfy the assumptions of the first parts of Theorems 1 and 2. Then as $T \rightarrow \infty$

$$
E \widehat{J_{4, T}}(\varphi) \rightarrow J_{4}(\varphi)
$$

II. Let the taper $\tilde{h}(t)$, the spectral densities $f_{2}, f_{4}$ and the function $\varphi$ satisfy the assumptions of the second parts of Theorems 1 and 2. Then

$$
E \widehat{J_{4, T}}(\varphi)-J_{4}(\varphi)=O\left(T^{-2}\right) \text { as } T \rightarrow \infty .
$$

Remark 1. In the case of observations over the domain $D_{T}=[1, T]^{d}$ Assumption 1 must be modified as follows: $\tilde{h}(t)$ is a positive measurable function of bounded variation with support on $[0,1]$ and $h(0)=0, h(1-v)=h(v)$ for $0 \leq v \leq \frac{1}{2}$.

Remark 2. Examples of tapers $\tilde{h}(t)$ satisfying the assumptions introduced in the discrete and continuous cases respectively are $\tilde{h}(t)=\frac{1}{2}(1+\cos (4 \pi t),|t| \leq 1$, and $\tilde{h}(t)=1-|t|,|t| \leq 1$.

Remark 3. We considered the case of the taper which factorizes: $h(t)=\prod_{i=1}^{d} \tilde{h}\left(t^{(i)}\right)$. This assumption simplifies calculations and allows to use known results for the one-dimensional case, namely, upper bounds for spectral windows $H_{k, T}(\lambda)$ and their convolution properties (see, e.g., Dahlhaus (1983)). For such type of tapers we also have that the approximate identity property of the corresponding Fejér-type kernels $\Phi_{k, T}^{h}$ is preserved for dimensions $\mathrm{d}>1$ (see Appendix). Additional assumptions on tapers help to achieve the rate $T^{-2}$ for the convergence of bias to zero, which is important for the spatial data. Note that without tapering or without some additional conditions on taper one can have only the rate $T^{-1}$.

Remark 4. The estimate (31) has the advantages over the estimates (8), (10), taken with $k=4$, since we do not have to introduce the restrictions on the domain of integration, and as a result we obtain the estimate of the functional $J_{4}(\varphi)$ itself. However, for the general $k$ the form of recursive estimates of this type becomes rather involved. The simple estimate can be still obtained for $k=5$, in which case from the integral of the fifth-order periodogram we should subtract some combination of the integrals of products of the periodograms of the second and third orders.

Remark 5. The functional (29) was used to construct the minimum contrast estimators for stationary processes in Sakhno (2012), in order to extend these estimators for the case of fields the result on the bias rate stated in Theorem 3 is of importance.

Remark 6. The consistency of the considered spectral estimates can be stated by using the same approach as in the papers Anh et al. (2004), Anh, Leonenko, and Sakhno (2007a), Anh et al. (2007b), variance of the estimators can be represented by the integrals involving the corresponding higher-order Fejér-type kernels, and, in order to apply the approximate identity property of these kernels, appropriate conditions on spectral densities of up to the eighth order must be introduced. 


\section{Appendix}

We present here some tools needed for the proofs.

Firstly, we note that the following formula for the cumulants of the finite Fourier transform $d_{T}^{h}(\lambda), \lambda \in \mathbb{S}$, can be deduced:

$$
\begin{aligned}
\operatorname{cum}\left(d_{T}^{h}\left(\alpha_{1}\right), \ldots, d_{T}^{h}\left(\alpha_{k}\right)\right)= & \int h_{T}\left(t_{1}\right) \ldots h_{T}\left(t_{k}\right) e^{-i \Sigma_{1}^{k}\left(\alpha_{j}, t_{j}\right)} \\
& \times \operatorname{cum}\left(X\left(t_{1}\right), \ldots, X\left(t_{k}\right)\right) \nu\left(d t_{1}\right) \ldots \nu\left(d t_{k}\right) \\
= & \int_{\mathbb{S}^{k-1}} f_{k}\left(\gamma_{1}, \ldots, \gamma_{k-1}\right) \int h_{T}\left(t_{1}\right) \ldots h_{T}\left(t_{k}\right) e^{i \Sigma_{1}^{k-1}\left(t_{j}, \gamma_{j}-\alpha_{j}\right)} \\
& \times e^{i\left(t_{k},-\Sigma_{j=1}^{k-1} \gamma_{j}-\alpha_{k}\right)} \nu\left(d t_{1}\right) \ldots \nu\left(d t_{k}\right) d \gamma_{1} \ldots d \gamma_{k-1} \\
= & \int_{\mathbb{S}^{k-1}} f_{k}\left(\gamma_{1}, \ldots, \gamma_{k-1}\right) H_{1, T}\left(\gamma_{1}-\alpha_{1}\right) \ldots H_{1, T}\left(\gamma_{k-1}-\alpha_{k-1}\right) \\
& \times H_{1, T}\left(-\Sigma_{1}^{k-1} \gamma_{j}-\alpha_{k}\right) d \gamma_{1} \ldots d \gamma_{k-1},
\end{aligned}
$$

where $H_{1, T}(\lambda)=\int h_{T}(t) e^{-i(t, \lambda)} \nu(d t)$.

If $\sum_{j=1}^{k} \lambda_{j}=0$, and $H_{k, T}(0) \neq 0$, then

$$
\Phi_{k, T}^{h}\left(\lambda_{1}, \ldots, \lambda_{k-1}\right)=\left((2 \pi)^{d(k-1)} H_{k, T}(0)\right)^{-1} \prod_{j=1}^{k} H_{1, T}\left(\lambda_{j}\right)
$$

is a multidimensional kernel of Fejér type over $\mathbb{S}^{k-1}$, which is an approximate identity for convolution, and the following equality holds:

$\lim _{T \rightarrow \infty} \int_{\mathbb{S}^{k-1}} \Phi_{k, T}^{h}\left(u_{1}, \ldots, u_{k-1}\right) G\left(u_{1}-v_{1}, \ldots, u_{k-1}-v_{k-1}\right) d u_{1} \ldots d u_{k-1}=G\left(v_{1}, \ldots, v_{k-1}\right)$,

provided that the function $G\left(u_{1}, \ldots, u_{k-1}\right)$ is bounded and continuous at the point $\left(v_{1}, \ldots, v_{k-1}\right)$, for the case $\mathbb{S}=[-\pi, \pi)^{d}$, and is from $L_{1}\left(\mathbb{S}^{k-1}\right) \bigcap L_{k-2}\left(\mathbb{S}^{k-1}\right)$ and continuous at the point $\left(v_{1}, \ldots, v_{k-1}\right)$, for the case $\mathbb{S}=\mathbb{R}^{d}$. In the case under consideration, when the taper factorizes, and the domain of observation is a cube $D_{T}=[-T, T]^{d}$, this fact follows as a straightforward generalization of the corresponding results stated for dimension $d=1$ by Dahlhaus (1983) (for $\mathbb{S}=[-\pi, \pi)$ ) and Ginovyan and Sahakyan (2019) (for $\mathbb{S}=\mathbb{R}$ ). In the non-tapered case, when $h(t) \equiv 1$, this was shown in Bentkus (1972).

For the case when $\sum_{j=1}^{k} \alpha_{j}=0$, the formula (A.1) leads to the following expressions:

$$
\begin{aligned}
& \left((2 \pi)^{d(k-1)} H_{k, T}(0)\right)^{-1} \operatorname{cum}\left(d_{T}^{h}\left(\alpha_{1}\right), \ldots, d_{T}^{h}\left(\alpha_{k}\right)\right) \\
= & \int_{\mathbb{S}^{k-1}} \Phi_{k, T}^{h}\left(\gamma_{1}-\alpha_{1}, \ldots, \gamma_{k-1}-\alpha_{k-1}\right) f_{k}\left(\gamma_{1}, \ldots, \gamma_{k-1}\right) d \gamma_{1} \ldots d \gamma_{k-1} \\
= & \int_{\mathbb{S}^{k-1}} \Phi_{k, T}^{h}\left(u_{1}, \ldots, u_{k-1}\right) f_{k}\left(u_{1}+\alpha_{1}, \ldots, u_{k-1}+\alpha_{k-1}\right) d u_{1} \ldots d u_{k-1} .
\end{aligned}
$$

\section{References}

Anh V, Leonenko N, Sakhno L (2004). "Quasilikelihood-based Higher-order Spectral Estimation of Random Processes and Fields with Possible Long-range Dependence." Journal of Applied Probability, 41A, 35-54.

Anh V, Leonenko N, Sakhno L (2007a). "Minimum Contrast Estimation of Random Processes Based on Information of Second and Third Orders." Journal of Statistical Planning and Inference, 137(3), 1302-1331. 
Anh V, Leonenko N, Sakhno L (2007b). "Statistical Inference Using Higher-order Information." Journal of Multivariate Analysis, 98(4), 706-742.

Anh V, Leonenko N, Sakhno L (2010). "Evaluation of Bias in Higher-order Spectral Estimation." Theory of Probability and Mathematical Statistics, 80, 1-14.

Bentkus R (1972). "The Error in Estimating the Spectral Function of a Stationary Process." Liet. Mat. Rink., 12(1), 55-71.

Brillinger D, Rosenblatt M (1967). "Asymptotic Theory of Estimates of k-th Order Spectra." In: Harris, B. (ed.), Spectral Anal. Time Ser., New York: Wiley.

Dahlhaus R (1983). "Spectral Analysis with Tapered Data." Journal of Time Series Analysis, 4, 163-175.

Dahlhaus R, Künsch H (1987). "Edge Effects and Efficient Parameter Estimation for Stationary Random Fields." Biometrika, 74(4), 877-882.

Ginovyan M, Sahakyan A (2019). "Estimation of Spectral Functionals for Lévy-driven Continuous-time Linear Models with Tapered Data." Electronic Journal of Statistics, 13, $255-283$.

Guyon X (1995). Random Fields on a Network: Modelling, Statistics and Applications. Springer, New York.

Keenan D (1987). "Limiting Behavior of Functionals of Higher-order Sample Cumulant Spectra." Annals of Statistics, 15(1), 134-151.

Sakhno L (2007). "Bias Control in the Estimation of Spectral Functionals." Theory of Stochastic Processes, 29(1-2), 134-151.

Sakhno L (2011). "On the Estimation of Integrals of Higher-order Spectral Densities." Applied Statistics. Actuarial and Financial Mathematics, (1-2), 80-87.

Sakhno L (2012). "Minimum Contrast Estimation of Stationary Processes Based on the Squared Periodogram." Lithuanian Mathematical Journal, 50(4), 400-419.

Taniguchi M (1982). "On Estimation of the Integrals of the Fourth Order Cumulant Spectral Density." Biometrika, 69, 117-122.

\footnotetext{
Affiliation:

Lyudmyla Sakhno

Taras Shevchenko National University of Kyiv

Volodymyrska 64, 01601, Kyiv, Ukraine

E-mail: Ims@univ.kiev.ua
}

\section{Austrian Journal of Statistics}

published by the Austrian Society of Statistics

Volume 49

April 2020 http://www.ajs.or.at/

http://www.osg.or.at/

Submitted: 2019-12-15

Accepted: 2020-03-03 